H I G H L I G H T S

\section{Long live SIRT1}

Reporting in Cell, Leonard Guarente and colleagues provide a link between the NAD-dependent protein deacetylase SIRT1, forkhead transcription factor FOXO3a and mammalian longevity.

Previous work in Saccharomyces cerevisiae and Caenorhabditis elegans has shown that metabolic shifts increase the activity of SIR2 (known as Sir2 and SIR-2.1 in S. cerevisiae and C. elegans, respectively), which increases lifespan. In addition, in response to mutations in the insulin receptor, the activation of the C. elegans forkhead transcription factor DAF-16 is also known to increase longevity. However, although the data suggest that SIR2 requires DAF- 16 for longevity, the mechanism that connects these two proteins has remained unknown.

Now, Guarente and co-workers show that SIRT1, the mammalian SIR2 orthologue, binds to FOXO1, FOXO3a and FOXO4, which are all growth-factor-regulated forkhead transcription factors. In vitro, FOXO3a is acetylated by its co-factor p300, which increases the transcription of FOXO3a target genes, and this effect is enhanced under stress conditions including ultra-violet light or oxidative stress. SIRT1, on the other hand, deacetylates FOXO3a and represses its activity, even under stress conditions. SIRT1 also represses FOXO3a by binding to and deacetylating p300.

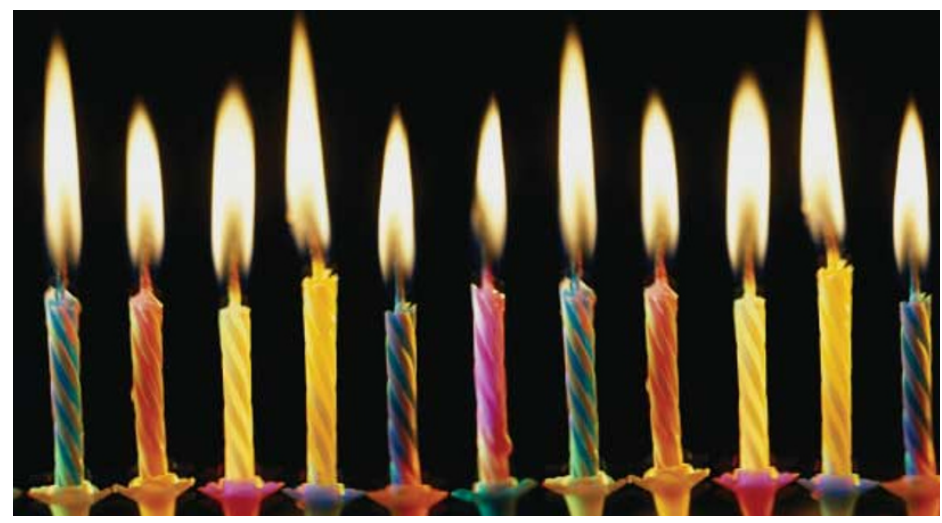

Guarente and colleagues confirmed their results in vivo, by showing that in $\mathrm{Sirt1}^{-/-}$mouse embryonic stem (ES) cells Foxo3a activity was increased, and that by adding Sirt 1 back to the cells the repression of Foxo3a was restored. In addition, in Sirt1 knockout mice the expression of the forkhead target genes phosphoenolpyruvate carboxykinase-1 (Pepck) and insulin-like growthfactor-binding protein-1 (Igfbp1) was increased. And p300, Foxo3a and Foxol were all shown to bind to the Foxo DNA-binding site in Sirt1 ${ }^{+/+}$ and Sirt $1^{-1}$ mice - Sirtl was also found to bind to the Foxo sites in Sirt1 $1^{+/+}$mice.

So, this work provides a link between two known regulators of lifespan in S. cerevisiae and C. elegans. Interestingly, though, the negative regulation of FOXO3a by SIRT1 in mammals seems to be opposite to the situation in C. elegans, in which it is the activation of DAF-16 that increases longevity.

But how does deacetylation of FOXO3a by SIRT1 repress its activity? The authors suggest that it might destabilize the protein, decrease its DNA-binding activity or alter its interactions with other proteins. The authors also speculate that the negative regulation of FOXO3a by SIRT1 in mammals, and the parallel repression of the tumour suppressor p53 also by SIRT1, could mitigate the effects of ageing by raising the threshold for apoptosis and cell senescence.

Natalie Wilson

(2) References and links ORIGINAL RESEARCH PAPER

Motta, C. M. et al. Mammalian SIRT1 represses (doi: 10.1016/S0092867404001266). WEB SITE

Leonard Guarente's laboratory: http://web.mit.edu/biology/guarente forkhead transcription factors. Cell 5 Feb 2004

\section{IN BRIEF}

\section{APOPTOSIS}

Quantitation of mitochondrial dynamics by photolabeling of individual organelles shows that mitochondrial fusion is blocked during the Bax activation phase of apoptosis.

Karbowski, M. et al. J. Cell Biol. 9 Feb 2004 (doi:10.1083/jcb.200309082)

A delicate balance between fusion and fission regulates the size, shape and number of mitochondria, but when this balance is altered in favour of fission, apoptosis occurs. Karbowski and colleagues describe a new technique - that uses a photoactivatable green fluorescent protein targeted to the mitochondrial matrix - to measure mitochondrial dynamics in vivo. Using this method, the authors show that mitochondrial fusion is blocked during apoptosis, and that this blockage could be caused by the activation of Bax or Bak.

\section{SIGNAL TRANSDUCTION}

APPL proteins link Rab5 to nuclear signal transduction via an endosomal compartment.

Miaczynska, M. et al. Cell 116, 445-456 (2004)

Miaczynska et al. have discovered a new signalling pathway that directly links the small GTPase Rab5, a regulator of endocytosis, with intracellular signalling. The pathway involves two Rab5 effectors, APPL1 and APPL2, which are concentrated in the endosomes. In response to external stimuli, APPL1 translocates to the nucleus and interacts with the NuRD-MeCP1 complex, which regulates gene expression. This work provides further evidence that endosomes aren't merely required for downregulating activated cell-surface receptors - they are also important for intracellular signalling.

\section{CELL ADHESION}

Localized stabilization of microtubules by integrin- and FAK-facilitated Rho signaling.

Palazzo, A. F. et al. Science 303, 836-839 (2004)

Integrins regulate Rac targeting by internalization of membrane domains.

del Pozo, M. A. et al. Science 303, 839-842 (2004)

Integrin signalling regulates numerous cellular processes, including cell adhesion and migration, and is mediated by Rho GTPases, such as Rho and Rac. Two studies now show that integrins use lipid rafts to target Rho and Rac to the plasma membrane and couple them to downstream effectors of the integrin-signalling pathway. del Pozo et al. found that Rac associates preferentially with lipid rafts, and the internalization of lipid rafts after cell detachment causes the loss of Rac membrane targeting and integrin signalling. Palazzo et al. report that the localized microtubule stabilization at the leading edge of migrating cells is mediated by integrin signalling, which regulates the local coupling of Rho to its effector Diaphanous (Dia) by the localization of lipid rafts at the leading edge. These findings implicate lipid rafts as spatial regulators in signalling events. 